\title{
The Stability of Lipid Rafts-Like Micro-Domains Is Dependent on the Available Amount of Cholesterol
}

\author{
Thi Thuy Minh Nguyen*, Vasudeva R. Chintamsetti, Sindhura Chennuru \\ Department of Chemistry and Biochemistry, Lamar University, Beaumont, TX, USA \\ Email: *ttnguyen15@lamar.edu
}

Received 6 July 2016; accepted 4 August 2016; published 8 August 2016

Copyright (C) 2016 by authors and Scientific Research Publishing Inc.

This work is licensed under the Creative Commons Attribution International License (CC BY). http://creativecommons.org/licenses/by/4.0/

(c) (i) Open Access

\begin{abstract}
Lipid rafts are sterol and sphingolipid rich membrane domains that possibly may play roles in multiple cellular processes. These domains are still the matter of debate and it is still unknown by which mechanism if any and organisms promote their formation. This study centers on the ease of in vitro formation of lipid rafts-like structures as it relates to the relative availability of sphingolipids, phospholipids, cholesterol, and membrane proteins. Following a $12 \mathrm{~h}$ incubation period, isolation and extraction of the lipid rafts-like assemblies, the composition of the structures was evaluated using HPLC. Cholesterol and sphingomyelin were detected at $206 \mathrm{~nm}$ and phosphatidylcholine was detected at $254 \mathbf{n m}$. Identification of lactose permease, a typical membrane protein, was done using FTIR. The thermal stability of the produced structures was also determined. Results show that the addition of cholesterol significantly increased both the amount of insoluble lipid rafts-like structures and their stability, and that the availability of a minimum amount of sphingolipid was necessary to produce larger amounts of more stable structures. However, the addition of phospholipids hindered the formation of lipid rafts-like assemblies and those formed were generally less stable.
\end{abstract}

\section{Keywords}

Lipid Rafts, Membrane Domains, Cholesterol

\section{Introduction}

The plasma membrane is mostly composed of loosely packed phospholipids capable of rapid lateral diffusion.

${ }^{*}$ Corresponding author.

How to cite this paper: Nguyen, T.T.M., Chintamsetti, V.R. and Chennuru, S. (2016) The Stability of Lipid Rafts-Like MicroDomains Is Dependent on the Available Amount of Cholesterol. Journal of Biophysical Chemistry, 7, 74-85. 
Liquid-ordered and disordered states can coexist on the same plasma membrane and those liquid-ordered domains can remain insular and maintain their relative rigidity among the neighboring phospholipids' bilayers [1] [2]. Such domains can, under proper circumstances of adequate amounts of cholesterol and sphingolipids, form into more stable structures within the membrane called lipid rafts [3]. Lipid rafts are therefore micro-domains within cell membranes that are enriched in cholesterol and sphingolipids. These assemblies can be separated and identified because they are components of the membranes that are resistant to solubility using detergent.

Lipid rafts are known to incorporate larger amounts of various signaling molecules, such as cell surface receptors and intracellular signaling molecules, and thus, these micro-domains have been associated with many cellular functions [4]. They may play important roles in the regulation of receptor-mediated cellular function, and accordingly, the disruption of lipid rafts results in the impairment of signaling events and receptor function [5]. Some studies have suggested that lipid rafts may even play an important role in tumor cell progression and control [4] [6]. Other functions have been attributed to lipid rafts such as pathogen uptake, endocytosis, and intracellular trafficking [6] [7]. Lipid rafts and caveolae, a subset of lipid rafts characterized by the presence of the protein caveolin, have been implicated in numerous signaling pathways that regulate the proper functioning of the cardiovascular system [7]. The formation and mechanism of action of these micro-domains are unclear. Signaling molecules, which have been shown to be associated with lipid rafts [7], may be permanently localized in the micro-domains where they assemble into preformed signaling complexes, as in the case of caveolae. This system would facilitate and accelerate the response to extracellular stimuli. The possibility also exists that molecules of the same pathway may be segregated into different subsets of lipid rafts which would interact when these micro-domains are formed.

The chemical composition of lipid rafts may influence their role. Different investigations note that the number and nature of lipid rafts vary in different types of cells in the body. Cancerous cells were shown to contain a higher number of lipid rafts compared to normal cells. However, following the addition of a cholesterol depleting agent, methyl $\beta$-cyclodextrin, cell apoptosis was observed. When cholesterol was replenished an increase in the number of lipid rafts was noted. This effect was found to be more important on cancer cell lines compared to healthy cells. The viability of the cells was restored upon addition of cholesterol. On the other hand, depletion of cholesterol caused lipid raft disruption, and as a consequence lipid rafts were rendered nonfunctional [8]-[10]. Hence, a clear correlation seems to exist between the number of viable lipid rafts in cell membranes and the amount of available cholesterol.

At this time there are still debates on the role of lipid rafts in the cellular membrane [4]-[7] and perhaps more importantly, on the mechanisms by which lipid rafts formation is controlled by the cell. One of the main reasons causing the controversy is the difficulty to observe lipid rafts directly in vivo. Some techniques have been used to attempt to identify lipid rafts in live cells: photonic force microscopy, fluorescence resonance energy transfer, single fluorophore tracking microscopy and chemical cross linking. Other techniques can be used to identify rafts in disrupted cells, including flotation of detergent resistant membranes, antibody patching and immunefluorescence microscopy, and immune-electron microscopy [11]-[13]. However, none of these methods were used conclusively to determine the processes of lipid raft formation.

Toulmay and Prinz investigated what initiated the formation of these micro-domains [14]. They observed that glucose scarcity, protein synthesis interruptions, and low $\mathrm{pH}$ induced the formation of vacuole, i.e. lipid rafts assemblies. Increases in $\mathrm{pH}$ failed to show this effect. Toulmay and Prinz also showed that lipid raft domains, of larger dimension than first anticipated, could form in vivo. Georg et al. reported on some investigations which demonstrated that sphingolipids showed stronger affinity towards cholesterol than phospholipids, and on studies that suggested the interactions between cholesterol and sphingomyelin were completely different from those interactions occurring between phosphatidylcholine and cholesterol [15]. Experiments performed on artificial membranes showed that lipids such as saturated phosphatidylcholines were able to form similar domains with cholesterol. Characterization of these structures by various physicochemical methods revealed that they formed a highly ordered and densely packed liquid ordered phase. The melting point of mixtures of phosphatidylcholine and cholesterol was also studied and the influence of ethanol and of cholesterol was evaluated. Wydro et al. showed that cholesterol increased the ordering of acylchains as well as the membrane surface density, while the effect of cholesterol on saturated chains varied from that found on unsaturated chains [11]. Cholesterol interactions with phosphatidylcholine and sphingomyelin did not follow a linear relationship as preferential interactions between sphingomyelin and cholesterol were detected [16]. This effect could be attributed to the denser packing 
of sphingomyelin molecules compared to the packing of phosphatidylcholine molecules [17].

All these studies demonstrate that membrane structure dynamics can be clearly understood only by the study of lipid interactions. Some Eggeling et al. argued that while somewhat flawed compared to in vivo studies, using liposome based techniques to study membranes physical properties may prove to be useful to develop better models of cellular behavior [18]. Studies of the physical properties of biological membranes have also been performed on model layers containing cholesterol induced liquid ordered phases [19]. Aggregation of liposomes due to the addition of ethanol was also investigated with the conclusion that the addition of ethanol resulted in increased fusion of the liposomes [20]. The permeability of lipid vesicles made of dipalmitoylphosphatidylcholine was found to increase by 8 times when cholesterol was added by a factor of $25 \%$ more compared to vesicles without cholesterol [21]. Other physical properties were investigated and the modulus of micro-domains was shown to significantly change with the addition of cholesterol, suggesting a strong dependence on the assembly [22]. Lingwood and Simons reviewed the potential of these self-assembled domains to combine and/or attract specific proteins and promote selective cellular processes [23]. Observations using atomic force microscopy demonstrated that ethanol influenced the bilayer properties according to membrane lateral organization [24]. Calorimetric investigations of mixtures of ethanol and liposomes were also performed, and the results obtained showed that the addition of cholesterol in lower concentrations helped to increase the interactions, while cholesterol in higher concentrations helped reduce the interactions [8] [25].

The physical properties of liposomes have been studied; however, the formation of insoluble micro-domains within liposomes has not. In addition, the lipid rafts hypothesis assumes that these micro-domains have an important property, i.e. they can include or exclude proteins selectively. Therefore, the investigation of artificial membranes should incorporate membrane proteins. Together, these would more accurately represent the in vivo formation of lipid rafts. Hence the first step in understanding not only the role of lipid rafts but the pathways to their formation is to study the growth and assembly of these domains. An in vitro investigation of each individual component involved in lipid rafts may shed light on the topic. This paper reports on the in vitro systematic investigation of the effect of the variation of the four most important components of lipid rafts—phospholipids, sphingolipids, cholesterol, membrane proteins—on the formation of detergent insoluble micro domains within liposomes.

\section{Methodology}

\subsection{Procedure for the Preparation of Lipid Rafts}

The preparation and analysis of lipid rafts involved several steps. The following is the generalized method used for the preparation of lipid raft and is followed for all sample preparations.

Cholesterol, sphingomyelin, phosphatidylcholine and proteins were mixed in test tubes in different concentrations. Systematic series of mixtures involved varying the concentration of one component while maintaining the concentrations of all other compounds constant at $3.0 \mathrm{mg} / \mathrm{mL}$. After mixing, these solutions were kept in a water bath at $37^{\circ} \mathrm{C}$ for $24 \mathrm{hrs}$. During this incubation period the components are expected to self-assemble. This preparation was kept under refrigerated conditions at $4^{\circ} \mathrm{C}$ for $6 \mathrm{hrs}$. The refrigerated solution was then taken and kept aside to reach room temperature, $25^{\circ} \mathrm{C}$. This solution was distributed in equal amounts to $1.5 \mathrm{~mL}$ Eppendorf tubes and centrifuged at 14,000 rpm for $30 \mathrm{~min}$. The components which were not associated in to the lipid rafts remained in the supernatant layer while the membrane assemblies formed a pellet. The supernatant solution was separated and saved. To the precipitate $200 \mu \mathrm{L}$ of pure methanol was added and kept for drying for about 30 min under nitrogen gas. The lipid raft assemblies were extracted by the addition of detergent [26] [27] and further centrifugation. The insoluble component contained the lipid raft assemblies. To the dried concentrate, $200 \mu \mathrm{L}$ of pure methanol were added. All the samples were subjected to HPLC for the quantification of cholesterol, phosphatidylcholine and sphingomyelin. For the quantification of the transmembrane protein, the samples were subjected to ATR-FTIR.

\subsection{Materials}

Cholesterol was purchased from Mallinckrodt Baker Inc. (Paris, Kentucky, USA) and was used without any further processing. Phosphatidylcholine was obtained from Aldrich. Sphingomyelin was bought from Fox Scientific, Inc. while the proteins were purchased from Bio-Rad. 


\subsection{Analysis Techniques}

High performance liquid chromatography (HPLC) was mainly used to separate and identify the components of the self-assemblies. A Waters HPLC including at $250 \mathrm{~mm}$ C18 column with 2487 dual wavelength absorbance detector, 1525 binary HPLC pump, 717 plus auto sampler and a Rheodyne manual injector were used. The operation of the instrument, integration of the peaks and the area calculations were done using the Breeze software. Cholesterol and sphingomyelin were detected at $206 \mathrm{~nm}$ and phosphatidylcholine was detected at $254 \mathrm{~nm}$. The retention time of cholesterol, phosphatidylcholine and sphingomyelin was obtained at around $30 \mathrm{~min}, 8.6 \mathrm{~min}$ and 9.3 min respectively.

A Thermo-Electron Nexus 470 Fourier transform infrared (FTIR) spectrophotometer in ATR (Attenuated Total Reflectance) mode was used to evaluate the structural characteristics and quantification of the protein components in the lipid rafts-like structures. The amide II band at $1456 \mathrm{~cm}^{-1}$ was used to quantify the amount of protein by comparison with calibration curves of pure lactose permease protein. Each sample was analyzed individually using a resolution of $4 \mathrm{~cm}^{-1}$. Baseline correction calculations were determined using the FTIR Omnic software package.

The liposomes formed from the self-assembled complexes containing phosphatidylcholine and sphingomyelin were directly observed using an Olympus BX51 polarized light microscope enhanced with a $530 \mathrm{~nm}$ filter equipped in color differentiation and contrast optimization. An Olympus U-CMAD3 camera attachment and QC Capture Pro 6.0 software allowed us to view and collect images.

\section{Results and Discussion}

Lipid rafts are predominantly composed of sphingolipids, sterol molecules, somephospholipids, and an array of transmembrane proteins. At this time, it is unclear what initiates the formation of lipid rafts. Phospholipids and sphingolipids can self-assemble and incorporate other molecules, however, this process would be random. It seems more likely that cells control the formation of nano-domains involved in other processes. The objective of this work is therefore to understand the composition and more importantly, the initiation process of the formation of lipid rafts in cellular membranes. Prior studies have suggested the existence of interactions between cholesterol and sphingolipids (9). As cholesterol binds in-between two sphingomyelin molecules the assembly is rigid and more resistant to solvation, hence an increase in sphingolipids and a minimum amount of cholesterol may lead to lipid raft formation. A series of experiments were designed based on the hypothesis that the self-assembly of domains involving sphingolipids is initiated by the occurrence of specific amounts of all components and of sterols in particular. The implications are that the rate of lipid raft formation could be increased dramatically by adjusting the relative amounts of lipid raft components. This hypothesis, if verified, implies that the cellular organism can control the amount of lipid rafts by synthesizing more or less of these components in the correct ratio. To test the hypothesis in vitro simulations of lipid rafts-like domains were formed and investigated. Solutions containing mixtures of the basic components of lipid rafts were prepared and incubated to allow time for the compounds to interact, after which any lipid rafts-like assemblies formed were isolated and analyzed using HPLC and FTIR spectroscopy. The basic components used were phosphatidylcholine and sphingomyelin, typical phospholipids and sphingolipids respectively, and cholesterol. Figure 1 shows the general molecular structures of this compound. Proteins were also added as a mixture containing a large fraction lactose permease, a membrane protein.

\subsection{Composition of Micro-Domains}

The compositional changes in the lipid rafts organized in the presence of lactose permease proteins were tested using four systematic variations. The first deals with the effect of increasing the amount of cholesterol on the composition of the in vitro lipid rafts. The second and third address the changes in the composition of lipid raftlike assembliesas the amounts of phosphatidylcholine and sphingomyelin are systematically increased. The last variation deals with the addition of the lactose permease proteins. In all variations, the same methodology was applied; in vitro lipid rafts were allowed to form from solutions of the four components of lipid rafts by keeping the concentration of three components constant and varying the concentration of the fourth. Following incubation and separation of the lipid raft-like assemblies through the use of detergents and centrifugation, the effect of the increase in concentration of this fourth component on the amount and composition of the lipid rafts was determined. 


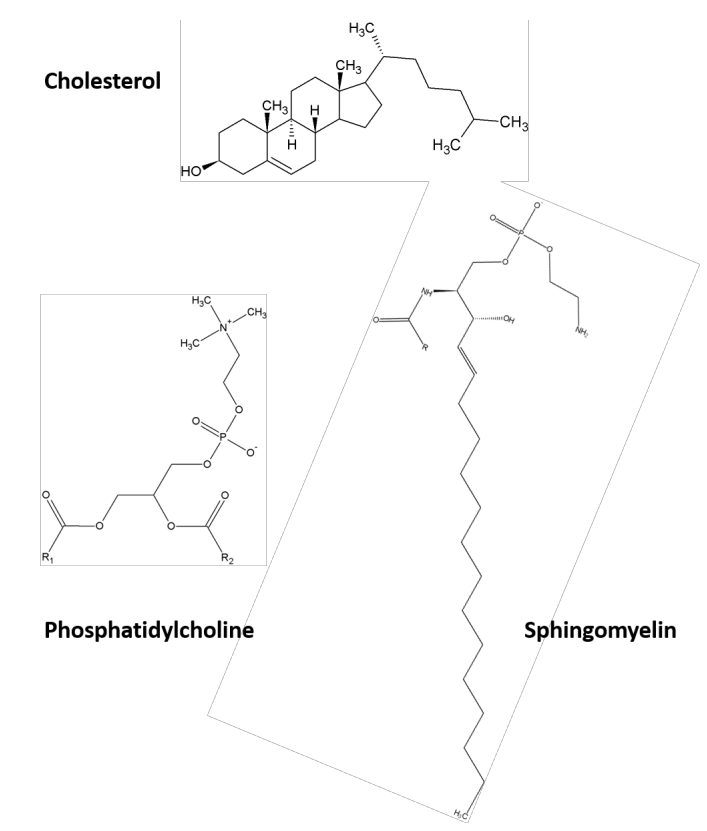

Figure 1. General molecular structures of cholesterol, phosphatidylcholine, and sphingomyelin, the three main regular components of cellular membranes in addition to proteins.

In the first set of experiments, self-assembled structures were allowed to form from solutions where the concentration of cholesterol was varied as stated in the methodology section, while the concentrations of phosphatidylcholine, sphingomyelin and lactose permease proteins were held constant. Following the incubation time and extraction, the lipid-rafts-like structures were extracted and analyzed using HPLC. The areas under the peaks associated with the three lipid components were converted into concentrations. Protein analysis was obtained using FTIR.

Figure 2 presents the change in the amounts of cholesterol, phosphatidylcholine, sphingomyelin, and lactose domains as the initial concentration of cholesterol is increased. A positive and quite linear correlation is obpermease composing the extracted micro-domains as the amount of cholesterol is increased. Variations in the absolute concentrations are presented. Analysis of the relative amounts did not provide significant additional insight and are not presented here. The square markers represent the amount of cholesterol in isolated lipid raft served, suggesting a direct association of sterol molecules with micro-domains. Upon first hypothesis, cholesterol may act as a binding molecule between other components particularly sphingolipids, which could lead to increases in the lipid raft formation as the amount of cholesterol is increased. The concentrations shown in the figure reveal that more than $50 \%$ of the initial cholesterol amount participates in the formation of in vitro lipid rafts-like structures supporting the importance of sterols. The evolution of the amount of phosphatidylcholine within the micro-domains is represented by the diamond markers in Figure 2. A minimum amount is always encountered as these molecules readily self-assemble. As the amount of available cholesterol increases, the amount of phospholipids in the assembly increases but at a lesser rate. On the other hand, the amount of sphingomyelin, indicated by the triangle markers in Figure 2, increases following a sigmoidal curve. Low amounts of cholesterol result in a very large increase in the amount of sphingolipids in the micro-domains. As these molecules become part of the assemblies, the concentration of available sphingomyelin is reduced leading to a decrease in the effect of adding cholesterol. Figure 2 also shows that the amount of lactose permease proteins (round markers) involved in the micro-domains is negligible at low amounts of available cholesterol, but once a certain critical cholesterol concentration is achieved, the amount of proteins included in the micro-domains increases exponentially.

In a second set of experiments, lipid rafts-like structures were allowed to form and were extracted from solutions where the concentration of phosphatidylcholine was varied while the concentrations of cholesterol, sphingomyelin, and lactose permease were held constant. Following the incubation time and extraction, lipid-rafts-like 


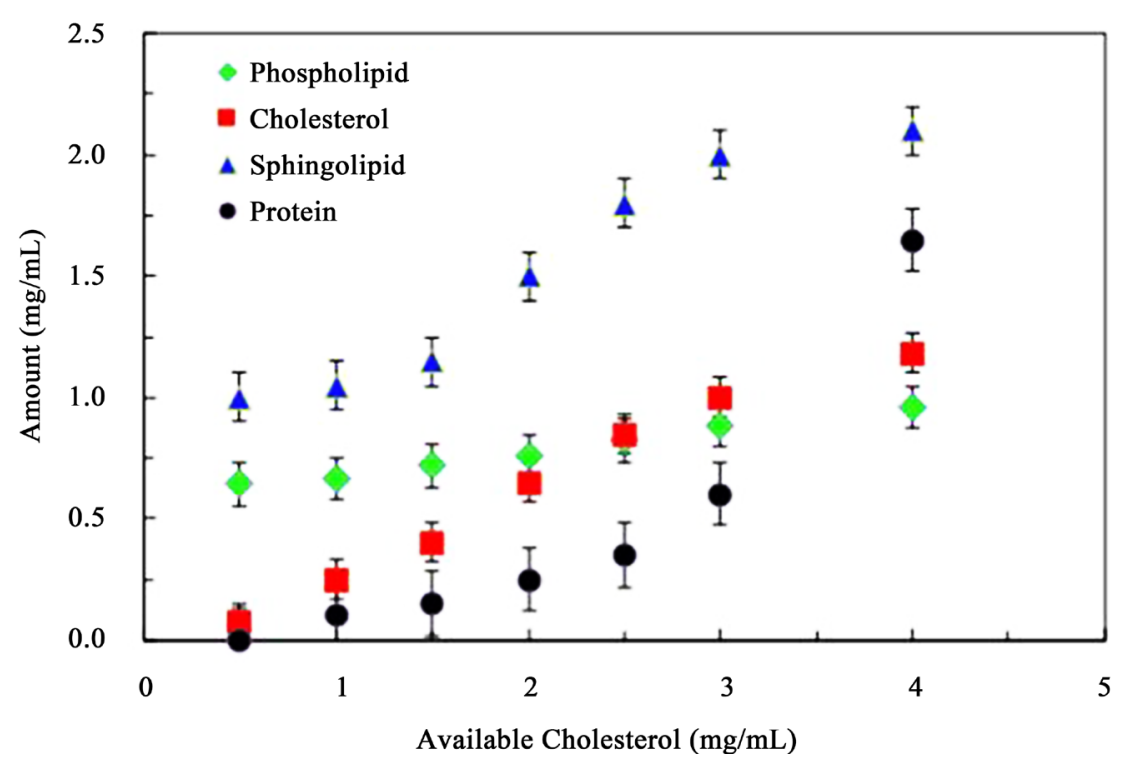

Figure 2. Molecular composition of lipid rafts-like micro-domains formed from solutions containing constant concentrations of phosphatidylcholine, sphingomyelin and lactose permease protein, and increasing concentrations of cholesterol.

micro-domains were analyzed using HPLC and infrared spectroscopy. Once again the areas under the peaks associated with the components were converted into concentrations.

Figure 3 presents the amount of phosphatidylcholine in isolated lipid raft domains as the initial concentration of phosphatidylcholine is increased. The amount of phosphatidylcholine recovered from the micro-domains is represented by the diamond markers. The observed decreasing trend line implies that as the amount of phospholipids is increased, less lipid rafts-like structures are formed. The concentration of phosphatidylcholine in the extracted lipid rafts is about 30 times lower than the initial solution concentration confirming the idea that phospholipids are not a primary component of lipid rafts. Figure 3 also shows the variation of the amount of cholesterol (square markers) and sphingomyelin (triangles) in isolated lipid raft-like domains as the initial concentration of phosphatidylcholine is increased. An increase in the concentration of phosphatidylcholine results in a decrease in the concentration of both cholesterol and phospholipids involved in the lipid raft formation supporting the argument that increasing the amount of phospholipids reduces the formation of lipid rafts-like micro-domains, most likely in favor of more detergent soluble structures. In all these structures, no significant amounts of lactose permease proteins were detected and these are not shown in the figure.

Lipid-rafts-like structures were then allowed to form and were extracted from solutions where the concentration of sphingomyelin was varied while the concentrations of cholesterol, phosphatidylcholine and lactose permease proteins were held constant. Figure 4 presents the results from these experiments. The amount of sphingomyelin isolated from lipid rafts-likemicro-domains as the initial concentration of sphingomyelinis increased is shown as triangle markers. A distinct increase in the concentration of sphingomyelin in the lipid rafts domains is observed: the concentration of sphingomyelin in the isolated lipid rafts-like structures significantly increased compared to the initial concentration suggesting that the number of lipid rafts increased as the amount of sphingolipids increased. This result is expected considering the fact that lipid rafts contain larger amounts of sphingolipids [2]. Figure 4 also shows the variation of the amount of cholesterol (square markers) in isolated lipid raft domains as the initial concentration of sphingomyelin is increased. At low concentrations of sphingomyelin, a constant amount of cholesterol is found implying that a minimum quantity of cholesterol is required for lipid raft formation. As the concentration of sphingomyelin increases, the amount of cholesterol follows a linear increase. One notes that the concentration of cholesterol is comparatively low even at higher sphingomyelin concentrations. This is attributed to the negative influence of the presence of phospholipids on lipid raft formation. These results suggest that a proper ratio of all lipid rafts components is needed for optimum lipid rafts formation. This argument may be further supported by the last component of Figure 4 as it shows the variation of the amount of phosphatidylcholine (diamond makers) in isolated lipid rafts-like micro-domains as the initial concentration of 


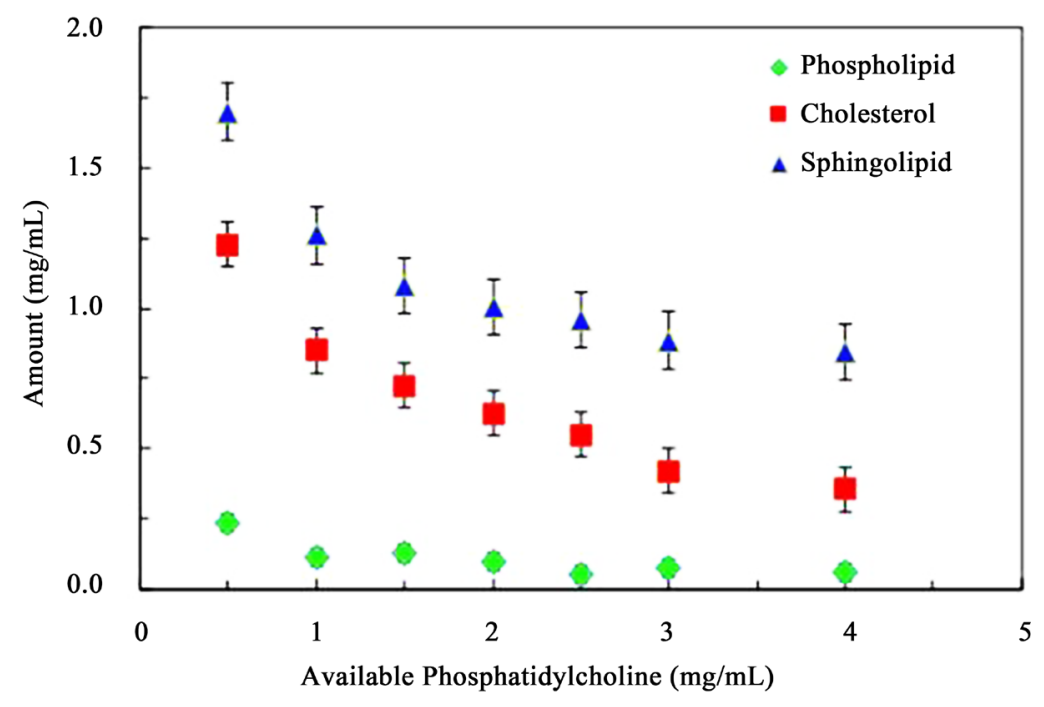

Figure 3. Molecular composition of lipid rafts-like micro-domains formed from solutions containing constant concentrations of, cholesterol, sphingomyelin and lactose permease protein, and increasing concentrations of phosphatidylcholine.

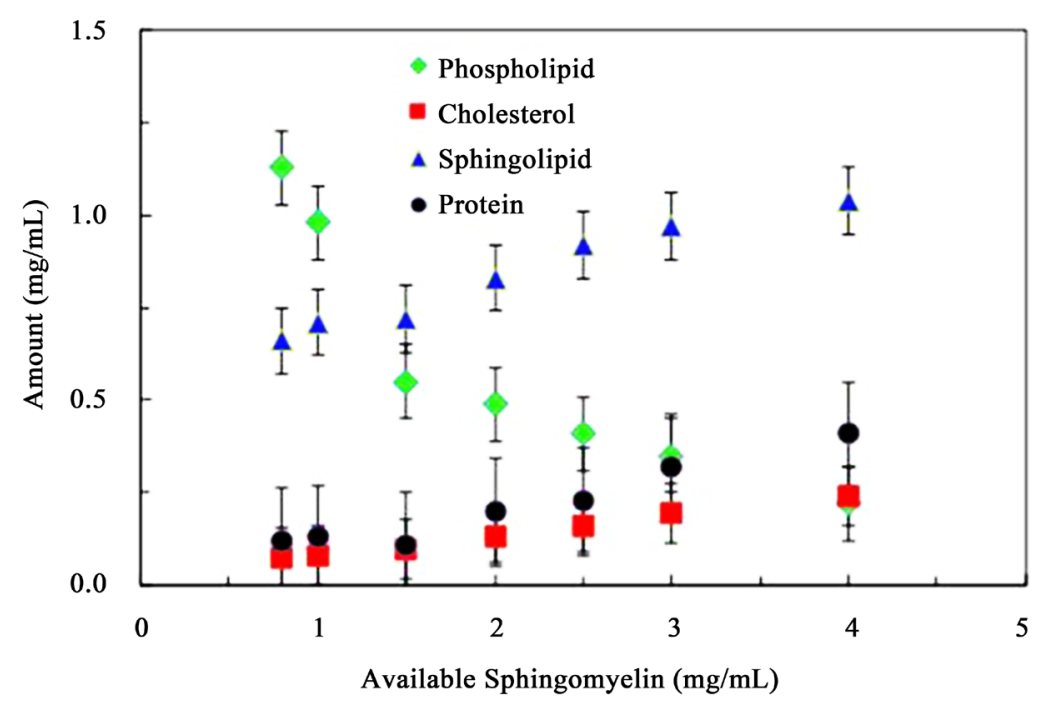

Figure 4. Molecular composition of lipid rafts-like micro-domains formed from solutions containing constant concentrations of phosphatidylcholine, cholesterol, and lactose permease protein, and increasing concentrations of sphingomyelin.

sphingomyelin is increased. A sharp decrease was observed suggesting that sphingomyelin replaces phosphatidylcholine in the extracted assemblies.

The change in the association of cholesterol, sphingomyelin, and phosphatidylcholine for the formation of the lipid rafts-like assemblies with the change in lactose permease protein was also tested. The concentration of cholesterol, sphingomyelin, and phosphatidylcholine were maintained constant. Following incubation and extraction of the lipid rafts-like micro-domains the relative amounts of each component were quantified using HPLC and FTIR. Figure 5 presents the evolution of cholesterol (square markers), sphingomyelin (triangle), phosphatidylcholine (diamonds), and lactose permease proteins (round markers) in lipid rafts-like assemblies as the amount of lactose permease protein available increases. As expected the amount of lactose permease involved in lipid rafts increases as the amount available in solution increases. However, the increase is limited and a maximum value is obtained even at high concentration of available protein. As the concentration of lactose permease protein increases, the concentration of cholesterol that associates in the complex also increases and, 


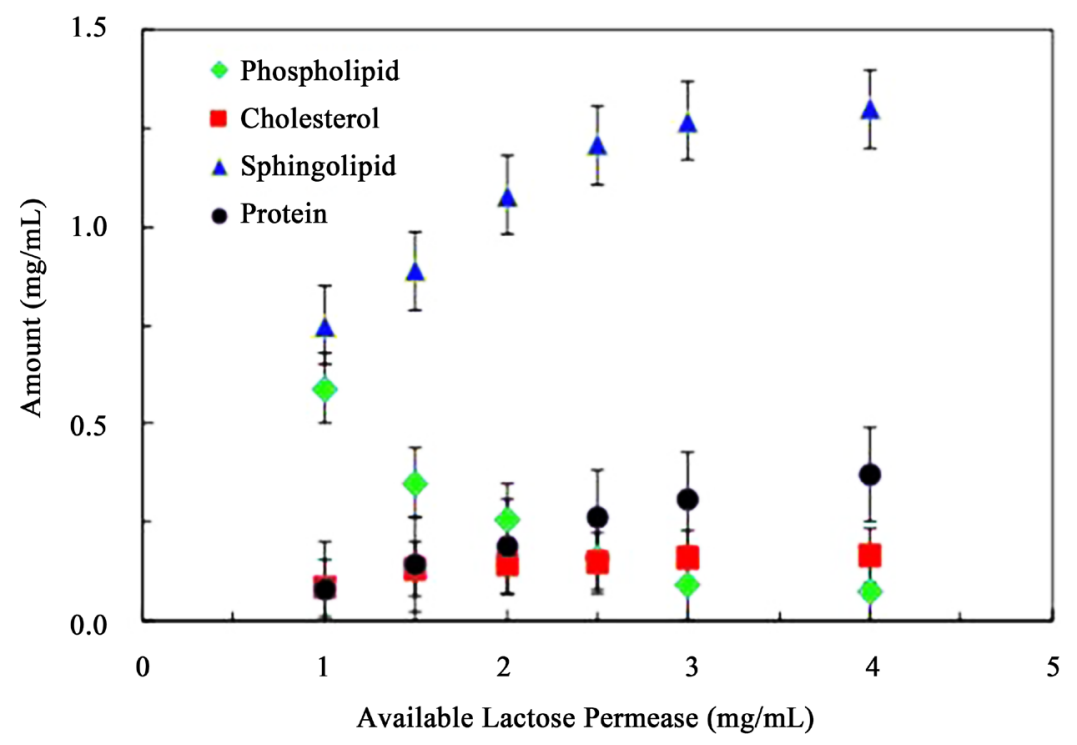

Figure 5. Molecular composition of lipid rafts-like micro-domains formed from solutions containing constant concentrations of phosphatidylcholine, cholesterol, and sphingomyelin, and increasing concentrations of lactose permease protein.

similar to the behavior of the protein, seems to tend towards a maximum value at high protein concentrations. The behavior is mirrored in the evolution of sphingomyelin amounts involved in the lipid rafts-like micro-domains. An increase towards a maximum value is observed. This is not the case with phosphatidylcholine. As the protein concentration increases the amount of phosphatidylcholine that participates in lipid rafts self- assembly decreases sharply indicating a negative correlation. Of further note is that the concentration plateaus observed in the behavior of the protein, cholesterol, and sphingolipid are reached at different amounts of available protein concentrations suggesting that the interdependent correlations between these types of molecules in different but important to the formation of lipid rafts-like structures. The quantifications of these intermolecular interactions within the micro-domain structures were evaluated using differential scanning calorimeter.

\subsection{Thermal Stability Measurements}

Differential scanning calorimetry (DSC) was used quantitatively evaluated the effect of the chemical composition on the overall stability of the lipid rafts-like assemblies. Figure 6 shows the typical DSC traces of samples of extracted micro-domains formed from mixtures of various amounts of phosphatidylcholine, and fixed concentrations of sphingomyelin, cholesterol and lactose permease in ethanol. At least 3 endotherms are observed at around $70^{\circ} \mathrm{C}, 80^{\circ} \mathrm{C}$ and $85^{\circ} \mathrm{C}$ respectively. These endotherms are associated with transitions related with the dissociation of the assemblies: a) a weakly bonded structure indicative of poorly assembled elements, b) a structurally sound assembly and c) a strongly bonded structure exhibiting greater stability. The temperature associated with the endotherms is related to the stability of the lipid rafts-like assemblies. Variations in this temperature would suggest the formation of more or less stable structures. To investigate if the composition influences the stability of the lipid rafts-like structures were formed and extracted from solutions containing different concentrations of phosphatidylcholine, sphingomyelin, cholesterol, and lactose permease. In Figure 6 the increase in the amount of available phosphatidylcholine clearly results in a general decrease in the temperature of all three endotherms indicating that the addition of this phospholipid results in the formation of less stable lipid rafts-like structures. This could be the effect of the insertion of the shorter phosphatidylcholine thereby reducing the stability of the structure. One also notes that the relative amounts of the higher temperature structures are also reduced as the phosphatidylcholine concentration is increased.

Quantification of all experiments was performed by plotting the temperature of the observed endotherms from the DSC traces as a function of concentration. Figure 7 presents the changes in the transition temperature of lipid rafts-like structures formed and extracted from solutions containing increasing concentrations of phosphatidylcholine, panel a, lactose permease, panel b, sphingomyelin, panel c, and cholesterol, panel d. In each panel, 


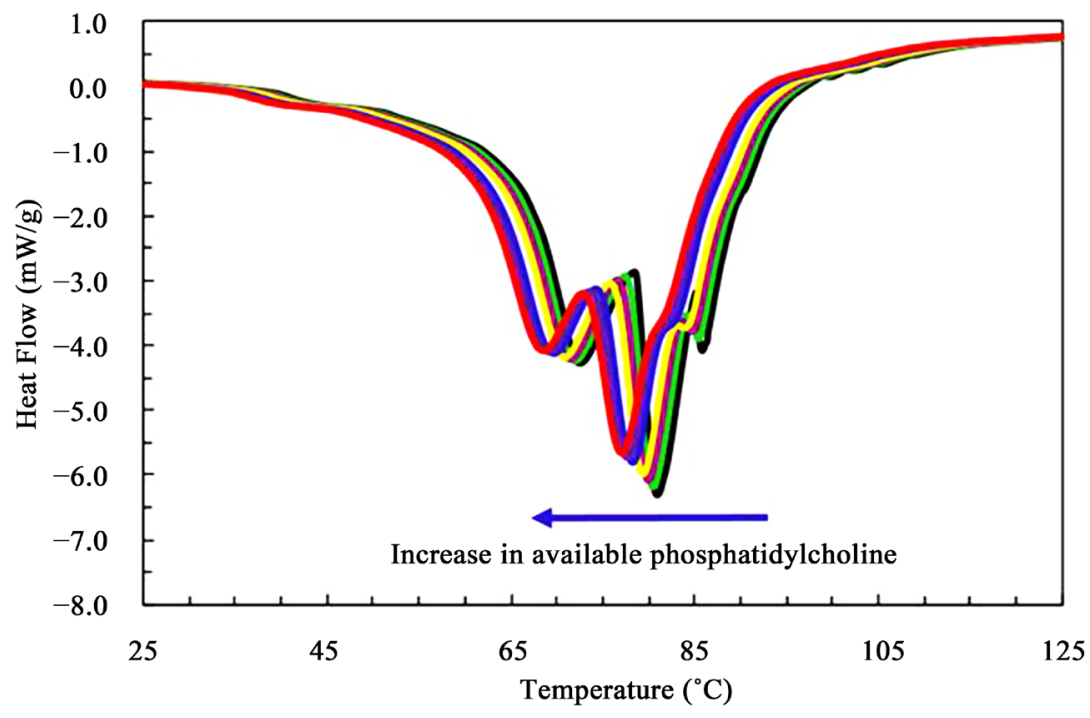

Figure 6. DSC trace of a typical in vitro lipid raft-like assembly. Three endotherms are clearly visible. See text for details.
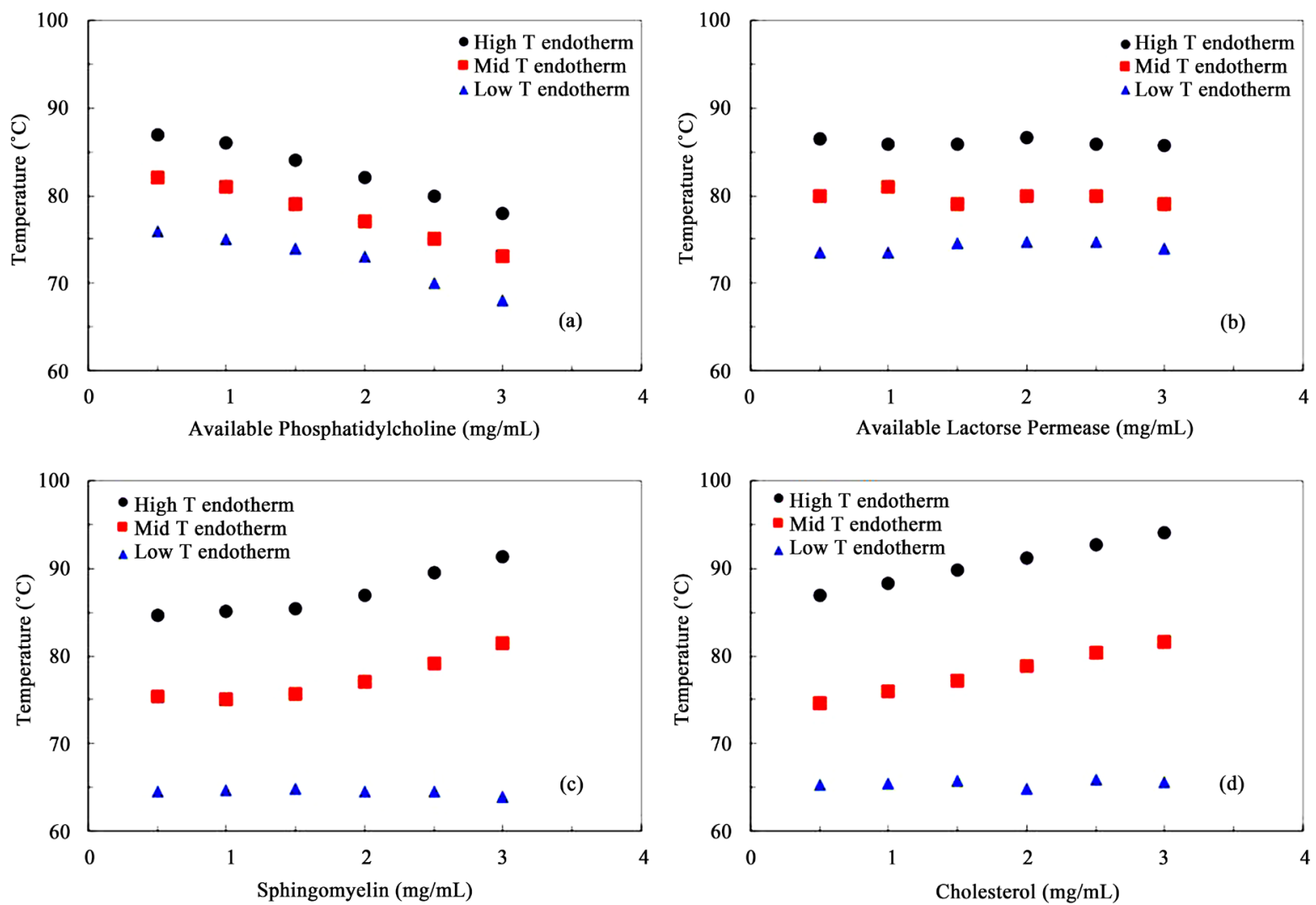

Figure 7. Thermal stability behavior of the lipid rafts-like micro-domains formed and extracted from solutions of different concentrations of compounds. Shown is the effect of varying the amount of available phosphatidylcholine (panel a), lactose permease (panel b), sphingomyelin (panel c), and cholesterol (panel d) while maintaining the concentration of the other three compounds constant at $3.0 \mathrm{mg} / \mathrm{mL}$.

the triangle indicate the low temperature endotherm observed around $70^{\circ} \mathrm{C}$, the square markers indicate the mid temperature endotherm at found around $80^{\circ} \mathrm{C}$ and the round markers in each panel represent the higher temperature endotherm at $85^{\circ} \mathrm{C}$. As expected from Figure 6, panel a of Figure 7 shows that as the amount of available 
phosphatidylcholine increases, the temperature of all three endotherm decreases indicating a loss in structural stability of the lipid rafts-like domains. Panel b shows that the addition of available protein does not significantly affect the transition temperature and therefore the stability of any structure. This suggests that the stability of the detergent insoluble lipid raft-like structures are not significantly related to the presence of membrane proteins, possibly because these compounds represent a low percentage of the relative composition of the assemblies. Figure 7 (c) shows the effect of the addition of sphingomyelin on the transition temperatures of the three transitions. Increasing the amount of available sphingolipids did not cause a variation in the transition temperature of the low temperature structure but it did result in a distinct increase in the temperature of both the mid and high temperature endotherms when the amount of available sphingomyelin reached a critical concentration around approximately $2.0 \mathrm{mg} / \mathrm{mL}$. For an organism to produce stable lipid rafts micro-domains a minimum amount of sphingolipids is therefore likely to be required. Figure 7 (d) also presents the effect of the concentration of available cholesterol on the stability of the assemblies. Once again, the low temperature endotherm does not seem affected by the concentrations of any of the constituents, suggesting that this transition is related to poorly formed assemblies that will dissociate readily. However, the amount of cholesterol has a significant impact on the transition temperature of the more stable structures. Increasing the available cholesterol by any amount results in an increase in the stability of the in vitro lipid rafts-like micro domains. This suggests that an optimum concentration of both sphingolipids and cholesterol can lead to the formation of lipid rafts. An organism could potentially control the amount of lipid rafts by modifying the amount of available cholesterol and by varying the ratio of the amounts of phospholipids and sphingolipids. This also leads to the possibility that if outside sources of these compounds are available, lipid rafts may form randomly which could lead to complications for the organism.

\section{Conclusion}

In vitro lipid raft-like membrane micro-domain was formed and extracted from solutions containing various concentrations of phosphatidylcholine, sphingomyelin, cholesterol and lactose permease membrane proteins, typical cellular membrane components. Quantification of the amounts of phosphatidylcholine, sphingomyelin, and cholesterol composing the detergent insoluble assemblies was performed using HPLC, while FTIR was used to evaluate the amount of proteins. Results indicate that increasing the amount of available sphingolipid and cholesterol leads to greater formation of lipid rafts-like assemblies. However, the increase in the relative components is only linear in the case of cholesterol. Thermal stability measurements using DSC further established that the assemblies were more stable if both sphingolipids and cholesterol's availability was high. In fact, a high amount of cholesterol and minimum concentration of sphingomyelin leads to the most stable structure. The amount of protein did not significantly affect the stability of the system while increasing the availability of phospholipids in solutions led to a decrease in the amount of lipid rafts-like structures and in the overall stability of any assemblies that were formed. Together, these results suggest that an organism could potentially induce the formation of lipid rafts by making available sufficient quantities of the key components, sphingolipids and cholesterol.

\section{Acknowledgements}

The partial support of the Welch Foundation (grant V-0004) is gratefully acknowledged.

\section{References}

[1] Bloch, K.E. (1983) Sterol Structure and Membrane Function. Critical Reviews in Biochemistry, 14, 47-92. http://dx.doi.org/10.3109/10409238309102790

[2] Brown, D.A. and London, E. (2000) Structure and Function of Sphingolipid and Cholesterol-Rich Membrane Rafts. The Journal of Biological Chemistry, 275, 17221-17224. http://dx.doi.org/10.1074/jbc.R000005200

[3] Fahy, E., Subramaniam, S., Brown, H.A., Glass, C.K., Merrill, A.H., Murphy, R.C., Raetz, C.R., Russell, D.W., Seyama, Y., Shaw, W., Shimizu, T., Spener, F., van Meer, G., VanNieuwenhze, M.S., White, S.H., Witztum, J.L. and Dennis, E.A. (2005) A Comprehensive Classification System for Lipids. The Journal of Lipid Research, 46, 839-861. http://dx.doi.org/10.1194/jlr.E400004-JLR200

[4] Li, Y.C., Park, M.J., Ye, S.K., Kim, C.W. and Kim, Y.N. (2006) Elevated Levels of Cholesterol-Rich Lipid Rafts in Cancer Cells Are Correlated with Apoptosis Sensitivity Induced by Cholesterol-Depleting Agents. American Journal 
of Pathology, 168, 1107-1118. http://dx.doi.org/10.2353/ajpath.2006.050959

[5] Simons, K. and Toomre, D. (2000) Lipid Rafts and Signal Transduction. Nature Reviews Molecular Cell Biology, 1, 31-39. http://dx.doi.org/10.1038/35036052

[6] Zhuang, L., Lin, J., Lu, M.L., Solomon, K.R. and Freeman, M.R. (2002) Cholesterol-Rich Lipid Rafts Mediate AktRegulated Survival in Prostate Cancer Cells. Cancer Research, 62, 2227-2231.

[7] Lucero, H.A. and Robbins, P.W. (2004) Lipid Rafts-Protein Association and the Regulation of Protein Activity. Archives of Biochemistry and Biophysics, 426, 208-224. http://dx.doi.org/10.1016/j.abb.2004.03.020

[8] Sezgin, E., Levental, I., Grzybek, M., Schwarzmann, G., Mueller, V., Honigmann, A., Belo, V.N., Eggeling, C., Coskun, U., Simons, K. and Schwille, P. (2012) Partitioning, Diffusion, and Ligand Binding of Raft Lipid Analogs in Model and Cellular Plasma Membranes. Biochimica et Biophysica Acta (BBA)—Biomembranes, 1818, 1777-1784. http://dx.doi.org/10.1016/j.bbamem.2012.03.007

[9] Mahammad, S., Dinic, J., Adler, J. and Parmryd, I. (2010) Limited Cholesterol Depletion Causes Aggregation of Plasma Membrane Lipid Rafts Inducing T Cell Activation. Biochimica et Biophysica Acta (BBA)—Biomembranes, 1801, 625-634. http://dx.doi.org/10.1016/j.bbalip.2010.02.003

[10] Kabouridis, P.S., Janzen, J., Magee, A.L. and Ley, S.C. (2000) Cholesterol Depletion Disrupts Lipid Rafts and Modulates the Activity of Multiple Signaling Pathways in T lymphocytes. European Journal of Immunology, 30, 954-963. http://dx.doi.org/10.1002/1521-4141(200003)30:3<954::AID-IMMU954>3.0.CO;2-Y

[11] Wydro, P., Flasinski, M. and Broniatowsk, M. (2013) Does Cholesterol Preferentially Pack in Lipid Domains with Saturated Sphingomyelin Over Phosphatidylcholine? A Comprehensive Monolayer Study Combined with Grazing Incidence X-Ray Diffraction and Brewster Angle Microscopy Experiments. Journal of Colloid and Interface Science, 397, 122-130. http://dx.doi.org/10.1016/j.jcis.2013.01.060

[12] Djoudi, F.A., Podechard, N., Collin, A., Chevanne, M., Provost, E., Poul, M., le Hegarat, L., Cathelin, D., Legrand, P., Therese, M., Boitrel, D., Gossmann, D.L. and Sergent, O. (2013) A Role for Lipid Rafts in the Protection Afforded by Docosahexaenoic Acid Against Ethanol Toxicity in Primary Rat Hepatocytes. Food and Chemical Toxicology, 60, 286-296. http://dx.doi.org/10.1016/j.fct.2013.07.061

[13] Temelli, F., Cordoba, A., Elizondo, E., Sarabia, M.C. and Veciana, J. (2012) Phase Behavior of Phytosterols and Cholesterol in Carbon Dioxide-Expanded Ethanol. Journal of Supercritical Fluids, 63, 59-68. http://dx.doi.org/10.1016/j.supflu.2011.12.012

[14] Toulmay, A. and Prinz, W.A. (2013) Direct Imaging Reveals Stable, Micrometer-Scale Lipid Domains that Segregate Proteins in Live Cells. The Journal of Cell Biology, 202, 35-44. http://dx.doi.org/10.1083/jcb.201301039

[15] Borner, G.H.H., Sherrier, D.J., Weimar, T., Michaelson, L.V., Hawkins, N.D., Askill, A.M., Napier, J.A., Beale, M.H., Lilley, K.S. and Paul Dupree, P. (2005) Analysis of Detergent-Resistant Membranes in Arabidopsis. Evidence for Plasma Membrane Lipid Rafts. Plant Physiology, 137, 104-116. http://dx.doi.org/10.1104/pp.104.053041

[16] Bach, D., Borochov, N. and Wachtel, E. (2002) Phase Separation of Cholesterol and the Interaction of Ethanol with Phosphatidylserine-Cholesterol Bilayer Membranes. Chemistry and Physics of Lipids, 114, 123-130. http://dx.doi.org/10.1016/S0009-3084(01)00189-X

[17] Mattijus, P. and Slotte, J.P. (1996) Does Cholesterol Discriminate between Sphingomyelin and Phospha-Tidylcholine in Mixed Monolayers Containing Both Phospholipids? Chemistry and Physics of Lipids, 81, 69-80. http://dx.doi.org/10.1016/0009-3084(96)02535-2

[18] Eggeling, C., Ringemann, C., Medda, R., Schwarzmann, G., Sandhoff, K., Polyakova, S., Belov, V.N., Hein, B., von Middendorff, C., Schönle, A. and Hell, S.W. (2009) Direct Observation of the Nanoscale Dynamics of Membrane Lipids in a Living Cell. Nature, 457, 1159-1162. http://dx.doi.org/10.1038/nature07596

[19] Teerawanichpan, P., Hoffman, T., Ashe, P., Datla, R. and Selvaraj, G. (2007) Investigations of Combinations of Mutations in the Jellyfish Green Fluorescent Protein (GFP) that Afford Brighter Fluorescence, and Use of a Version (VisGreen) in Plant, Bacterial, and Animal Cells. Biochimica et Biophysica Acta (BBA)—General Subjects, 1770, 13601368. http://dx.doi.org/10.1016/j.bbagen.2007.06.005

[20] Jury, E.C., Borja, F.F. and Kabouridis, P.S. (2007) Lipid Rafts in T Cell Signalling and Disease. Seminars in Cell \& Developmental Biology, 18, 608-615. http://dx.doi.org/10.1016/j.semcdb.2007.08.002

[21] Komatsu, H. and Okada, S. (1997) Effects of Ethanol on Permeability of Phosphatidylcholine/Cholesterol Mixed Liposomal Membranes. Chemistry and Physics of Lipids, 85, 67-74. http://dx.doi.org/10.1016/S0009-3084(96)02634-5

[22] Kim, K.H., Choi, S.Q., Zell, Z.A., Squires, T.M. and Zasadzinski, J.A. (2013) Effect of Cholesterol Nanodomains on Monolayer Morphology and Dynamics. Proceedings of the National Academy of Sciences of the United States of America, 110, E3055-E3060.

[23] Lingwood, D. and Simons, K. (2010) Lipid Rafts as a Membrane-Organizing Principle. Science, 327, 46-50. http://dx.doi.org/10.1126/science.1174621 
[24] Trandum, C., Westh, P., Jorgensen, K. and Mouristen, O.G. (1999) Association of Ethanol with Lipid Membranes Containing Cholesterol, Sphingomyelin and Ganglioside: A Titration Calorimetry Study. Biochimica et Biophysica Acta (BBA)—Biomembranes, 1420, 179-188. http://dx.doi.org/10.1016/S0005-2736(99)00092-9

[25] Marques, J.T., Viana, A.S. and De Almeida, R.F.M. (2011) Ethanol Effects on Binary and Ternary Supported Lipid Bilayers with Gel/Fluid Domains and Lipid Rafts. Biochimica et Biophysica Acta (BBA)—Biomembranes, 1808, 405414. http://dx.doi.org/10.1016/j.bbamem.2010.10.006

[26] Martosella, J., Zolotarjova, N., Liu, H., Moyer, S.C., Perkins, P.D. and Boyes, B.E. (2006) High Recovery HPLC Separation of Lipid Rafts for Membrane Proteome Analysis. Journal of Proteome Research, 5, 1301-1312. http://dx.doi.org/10.1021/pr060051g

[27] Chamberlain, L.H. (2004) Detergents as Tools for the Purification and Classification of Lipid Rafts. FEBS Letters, 559, $1-5$.

Submit or recommend next manuscript to SCIRP and we will provide best service for you:

Accepting pre-submission inquiries through Email, Facebook, LinkedIn, Twitter, etc.

A wide selection of journals (inclusive of 9 subjects, more than 200 journals)

Providing 24-hour high-quality service

User-friendly online submission system

Fair and swift peer-review system

Efficient typesetting and proofreading procedure

Display of the result of downloads and visits, as well as the number of cited articles

Maximum dissemination of your research work

Submit your manuscript at: http://papersubmission.scirp.org/ 